\title{
Risk Aversion over Finite Domains
}

\author{
Jean Baccelli*a, Georg Schollmeyer ${ }^{\dagger \mathrm{b}}$, and Christoph Jansen ${ }^{\ddagger c}$ \\ ${ }^{a}$ University of Oxford $\quad{ }^{b, c}$ Ludwig-Maximilians-Universität München
}

September 22, 2021

Abstract

We investigate risk attitudes when the underlying domain of payoffs is finite and the payoffs are, in general, not numerical. In such cases, the traditional notions of absolute risk attitudes, that are designed for convex domains of numerical payoffs, are not applicable. We introduce comparative notions of weak and strong risk attitudes that remain applicable. We examine how they are characterized within the rankdependent utility model, thus including expected utility as a special case. In particular, we characterize strong comparative risk aversion under rank-dependent utility. This is our main result. From this and other findings, we draw two novel conclusions. First, under expected utility, weak and strong comparative risk aversion are characterized by the same condition over finite domains. By contrast, such is not the case under non-expected utility. Second, under expected utility, weak (respectively: strong) comparative risk aversion is characterized by the same condition when the utility functions have finite range and when they have convex range (alternatively, when the payoffs are numerical and their domain is finite or convex, respectively). By contrast, such is not the case under non-expected utility. Thus, considering comparative risk aversion over finite domains leads to a better understanding of the divide between expected and non-expected utility, more generally, the structural properties of the main models of decision-making under risk.

Keywords: risk aversion; comparative risk aversion; expected utility; nonexpected utility; rank-dependent utility; dual expected utility

\section{JEL Classification Numbers: D 81}

\footnotetext{
*jean.baccelli@gmail.com (corresponding author)

${ }^{\dagger}$ georg.schollmeyer@stat.uni-muenchen.de

${ }^{\ddagger}$ christoph.jansen@stat.uni-muenchen.de
}

For helpful comments and suggestions, we thank two anonymous reviewers, Antoine Bommier, Federica Ceron, Simone Cerreia-Vioglio, Eric Danan, Lorenz Hartmann, Mark Machina, Marcus Pivato, as well as audiences at PROGIC 2019 and RUD 2020 We are especially grateful to Peter Wakker for his guidance in the early stages of the preparation of this paper. All errors and omissions are ours. Funding for this research was provided by the Ludwig-Maximilians-Universität München, the University of Pittsburgh, and the Deutsche Forschungsgemeinschaft (DFG project 426170771). 


\section{Introduction}

The traditional risk attitude concepts of economics are defined with reference to the more primitive notion of an increase in risk. The most widespread notion of an increase in risk is that of a mean-preserving spread (Rothschild and Stiglitz, 1970). This notion is usually introduced assuming that the underlying domain of payoffs is a convex subset of the reals, as is the case when the payoffs form a monetary interval, for instance. The resulting ideas of risk aversion, risk seeking, and risk neutrality have led to numerous applications in insurance theory, finance, and other areas of economics (e.g., Eeckhoudt et al., 2005). These model-free ideas can also be used to axiomatically analyze the structural properties of the main models of decision-making under risk. Specifically, they help better understand the fundamental divide between expected and non-expected utility. This is because risk attitudes turn out to be treated differently across this divide. In particular, one can define various logically nested kinds of increase in risk, accordingly, various degrees of risk aversion (or risk seeking), and subsequently prove that under expected utility, all degrees of risk aversion (or risk seeking) are characterized by the same condition, while such is not the case under non-expected utility (see esp. Chateauneuf et al., 1997).

However, many risky decisions are made with respect to finite domains of non-numerical payoffs. ${ }^{1}$ Economically relevant examples include nondivisible consumer goods, health conditions, or social positions, for instance. As the mean of an option then becomes a meaningless notion, it is not obvious how to define an increase in risk in those cases. Consequently, it is not obvious how to define risk attitudes. A coarse notion of increasing risk seems readily available, however. It is that (under suitable restrictions) any risky prospect is riskier than any riskless prospect, i.e., any payoff given with certainty. One can retrospectively interpret Yaari as building on that notion of increasing risk in his pioneering exploration (Yaari, 1969) of a comparative approach to risk aversion. In this approach, rather than the absolute notion "being risk averse", the central concept of interest becomes the more fundamental comparative notion "being more risk averse than". Importantly, comparative notions do not apply only when the domain of payoffs is finite and non-numerical, but also over convex real domains (e.g., Diamond and Stiglitz, 1974). However, in the former case, unlike in the latter, they turn out to be the only useful risk attitude notions available. The literature has now explored notions of increasing risk that are more refined than the coarse notion given above. The most important references are Allison and

\footnotetext{
${ }^{1}$ In a direction that is different from but complementary to the one explored here, one could also maintain the numerical structure of the domain of payoffs but generalize risk to uncertainty to investigate how to define an increase in uncertainty. See in particular Grant and Quiggin, 2005 and the references quoted therein. We are grateful to a referee for drawing our attention to Grant and Quiggin's paper, to which we briefly return in fn. 4 and 14 .
} 
Foster, 2004, Mendelson, 1987, and Bommier et al., 2012, with motivations coming from health economics, general statistics, and insurance economics, respectively. While only the first of these papers focuses on the finite case, the remaining two nonetheless also provide analytical tools that are applicable to that case. All three papers propose to define increasing risk by a simple single-crossing condition on the distribution functions associated to the options (see our Def. 1). Surprisingly, these three important contributions are independent of one another and - the last one excepted-Yaari's preexisting pioneering work. Overall, the current literature on risk attitudes over finite domains is not conceptually unified or systematically organized. Arguably it should, like the classic literature on absolute risk attitudes (e.g., Chateauneuf et al., 1997), be structured with reference to logically nested kinds of increasing risk.

Gathering insights from the above papers, we contribute to conceptually unifying the currently available notions of comparative risk attitudes over finite domains (see Secs. 3 and 4). In particular, building especially on Bommier et al., 2012 on the one hand and the classic literature on absolute risk attitudes on the other, we propose to distinguish between weak and strong comparative risk aversion (see Def. 2). We then investigate (in Sec. 5) the characterization of these notions in a large class of non-expected utility preferences, viz. the rank-dependent utility preferences (Quiggin, 1982). Such preferences include both expected utility and the so-called dual expected utility model (Yaari, 1987) as special cases. To the best of our knowledge, when applied to finite domains of payoffs, our notion of strong comparative risk aversion has not been hitherto characterized under expected utility, dual expected utility, or general rank-dependent utility. Building on Chateauneuf et al., 2005, we provide the missing characterizations (see Thm. 1, together with Cors. 1 and 2). Those are the main results in our paper. As regards our notion of weak comparative risk aversion, it has already been characterized under expected utility (Peters and Wakker, 1987), but not under dual expected utility or rank-dependent utility, to the best of our knowledge. We provide partial, yet instructive results on weak comparative risk aversion under dual expected utility (the non-necessity result in Obs. 2) and general rank-dependent utility (the sufficiency result in Obs. 3).

Furthermore, bringing together these new results and preexisting ones, we reach two novel conclusions (Facts 2 and 3). First, under expected utility, weak and strong comparative risk aversion are characterized by the same condition, which is not the case under non-expected utility. This conclusion holds not only when the utility functions have convex range, which was already known (e.g., Chateauneuf et al., 1997), but also when the utility functions have finite range, which had not been hitherto established. Second and more novel, under expected utility, weak (respectively: strong) comparative risk aversion is characterized by the same condition when the utility functions have finite range and when they have convex range (alternatively, 
when the payoffs are numerical and their domain is finite or convex, respectively). By contrast, such is not the case under non-expected utility. The latter kind of comparisons had not been hitherto studied in the literature, to the best of our knowledge. Accordingly, it is worth making immediately clear that it does not reduce to the former. Weak and strong comparative risk aversion could be characterized by the same condition both in the finite and in the convex case, yet the common condition differ across these two cases. Conversely, weak and strong comparative risk aversion could be characterized by different conditions both in the finite and in the convex case, yet the distinctive condition for each attitude remain the same across these two cases. The take-home message from our conclusions on these two distinct issues is that like absolute risk attitudes, comparative risk attitudes help better understand the fundamental divide between expected and nonexpected utility, more generally, the structural properties of the main models of decision-making under risk. We consider this general conceptual insight, which we explain to raise several interesting questions for further research, to be the main contribution of our investigation of risk attitudes over finite domains.

The rest of the paper is organized as follows. Sec. 2 gathers some necessary preliminaries. Sec. 3 presents the notion of increasing risk on which our analysis relies. Sec. 4 introduces our notions of weak and strong comparative aversion. Sec. 5 contains our main results and their discussion. Sec. 6 concludes.

\section{Preliminaries}

Let $X=\left\{x_{1}, \ldots, x_{n}\right\}$ be a finite set of payoffs. These payoffs may, but need not, be numerical, i.e., elements of $\mathbb{R}$; for our purposes, what matters is that there be, in any case, finitely many of them. Let $L=\Delta(X)$, with generic element $l=\left(p_{1}, x_{1} ; \ldots ; p_{n}, x_{n}\right)$, be the set of all probability distributions, or lotteries, over $X$. Let the preferences of the decision-maker be given by $\succcurlyeq$, a binary relation over $L$, with symmetric and asymmetric parts $\sim$ and $\succ$, respectively. We always assume that $\succcurlyeq$ is a weak order. Abusing notation as usual, $\succcurlyeq$ is also defined over $X$, identified with the set of degenerate lotteries in $L$. Notice that, by the above assumptions, the restriction of $\succcurlyeq$ to $X$ can be represented by a utility function $u: X \rightarrow \mathbb{R}$, the range of which is finite. That representation is ordinally unique. For non-triviality, we assume throughout that there are at least three payoffs between which the decision-maker is not indifferent, i.e., $x \succ y \succ z$ holds for some $x, y, z \in X$. Furthermore, we always assume that the elements of $X$ have been indexed consistently with their ranks in the decision-maker's preferences, i.e., $x_{1} \succcurlyeq \cdots \succcurlyeq x_{n}$ holds. Thus, given $l=\left(p_{1}, x_{1} ; \ldots ; p_{n}, x_{n}\right)$ and any $i, 1 \leq i \leq n, \sum_{j=1}^{i} p_{j}$ is the probability that lottery $l$ delivers a payoff $x$ at least as good as $x_{i}$, i.e., that 
$x \succcurlyeq x_{i}$.

In this paper, we focus on three models of decision-making under risk. Rank-dependent utility (RDU; Quiggin, 1982) holds if the following condition obtains. There exists a strictly increasing, continuous ${ }^{2}$ probability weighting function $w:[0,1] \rightarrow[0,1]$, with $w(0)=0$ and $w(1)=1$, and a utility function $u: X \rightarrow \mathbb{R}$, such that, with the convention $u\left(x_{n+1}\right)=0, \succcurlyeq$ is represented by the function $v: L \rightarrow \mathbb{R}$ given by:

$$
v(l)=\sum_{i=1}^{n}\left[w\left(\sum_{j=1}^{i} p_{j}\right)\left(u\left(x_{i}\right)-u\left(x_{i+1}\right)\right)\right] .
$$

In this representation, the utility function is cardinally unique and the probability weighting function is absolutely unique. Expected utility (EU) is the special case of (1) where, for all $p \in[0,1], w(p)=p$. When the elements of $X$ are numerical, dual expected utility (DEU; Yaari, 1987) is the special case of (1) where, for all $x \in X, u(x)=x$. Any model of decision-making under risk different from EU qualifies as a non-expected utility (non-EU) model.

For future reference, we also state now the definition of one core property satisfied by RDU and many other models of decision-making under risk. The relation $\succcurlyeq$ respects first-order stochastic dominance if for any $l, l^{\prime} \in L$, with $l=\left(p_{1}, x_{1} ; \ldots ; p_{n}, x_{n}\right), l^{\prime}=\left(q_{1}, x_{1} ; \ldots ; q_{n}, x_{n}\right)$, if $\sum_{j=1}^{i} p_{j} \geq \sum_{j=1}^{i} q_{j}$ holds for all $i, 1 \leq i \leq n$, and the inequality is strict for some $i$, then $l \succcurlyeq l^{\prime}$. Thus, if the cumulative distribution function of $l$ is nowhere higher, and somewhere strictly lower, than the cumulative distribution function of $l^{\prime}$, then $l \succcurlyeq l^{\prime}$.

Finally, we recall the characterization of concavity, which we here adopt as a definition, whereby a function $f: \mathbb{R} \rightarrow \mathbb{R}$ is concave (respectively: convex) if for any $a, b, c, d \in \mathbb{R}$ such that $a>b \geq c>d$, the inequality $(f(c)-f(d)) /(c-d) \geq(f(a)-f(b)) /(a-b)$ (respectively: the inequality $(f(a)-f(b)) /(a-b) \geq(f(c)-f(d)) /(c-d))$ holds. The function $f$ is strictly concave (respectively: convex) if this inequality is always strict. Furthermore, given two functions $f$ and $g$ that are real-valued (but not necessarily defined over real domains), we say that $f$ is more concave (respectively: more convex) than $g$ if there exists a strictly increasing concave (respectively: convex) function $h: \mathbb{R} \rightarrow \mathbb{R}$ such that $f=h \circ g$. The function $f$ is strictly more concave (respectively: convex) than $g$ if this transformation $h$ is strictly concave (respectively: convex).

\footnotetext{
${ }^{2}$ The literature has been more general in exploring discontinuities at 0 and 1 . Motivations have included accounting for the possibility effect, the certainty effect, and the like (e.g., Wakker, 2010, Chap. 6). For simplicity, we will assume these discontinuities away.
} 


\section{Increasing Risk}

The most fundamental definition in our paper is the following. As stated, it is (a slight variation on $)^{3}$ the definition proposed in Bommier et al., 2012.

Definition 1. Let $l=\left(p_{1}, x_{1} ; \ldots ; p_{n}, x_{n}\right)$ and $l^{\prime}=\left(q_{1}, x_{1} ; \ldots ; q_{n}, x_{n}\right)$ be two elements of $L$. Say that $l$ is a spread of $l^{\prime}$, noted here $l \vdash l^{\prime}$, if there exists a $k$, $2 \leq k \leq n$, such that for all $i \leq k-1, \sum_{j=1}^{i} p_{j} \geq \sum_{j=1}^{i} q_{j}$, and for all $i \geq k$, $\sum_{j=1}^{i} p_{j} \leq \sum_{j=1}^{i} q_{j}$, with at least one strict inequality in each direction.

Thus, $l \vdash l^{\prime}$ if the cumulative distribution functions of $l$ and $l^{\prime}$ single-cross, i.e., $l$ is first-order-stochastically dominated by $l^{\prime}$ on the left of the crossing point, then first-order-stochastically dominates it on the right of the crossing point. Therefore, appreciating all payoffs with reference to that point, $l$ makes the bad payoffs of $l^{\prime}$ worse, the good payoffs, better. ${ }^{4}$ The same fundamental intuition underlies the simplest kind of increase in risk in the classic convex real case (Diamond and Stiglitz, 1974; Machina, 1982; Machina and Pratt, 1997). A major difference is that the spread can then be calibrated as, e.g., mean-preserving, which is in general not possible in the finite case, where the mean of a lottery is, in general (specifically, when the payoffs are not numerical), a meaningless notion. This non-calibration notwithstanding, one can say that whenever $l \vdash l^{\prime}, l$ is riskier than $l^{\prime}$.

It is natural to single out the special case where $l \vdash l^{\prime}$ and $l^{\prime}$ is a degenerate lottery, i.e., a riskless payoff. This is when the above conditions hold with $\sum_{j=1}^{i} q_{j}=0$ for all $i \leq k-1$ and $\sum_{j=1}^{i} q_{j}=1$ for all $i \geq k$. Assuming the respect of first-order stochastic dominance, this is equivalent to the definition of increasing risk which - as a matter of retrospective attribution - one can naturally associate to the seminal exploration of risk attitudes in Yaari, 1969. We call such spreads basic. They are, from a logical point of view, the most restrictive kinds of increase in risk which one can define over finite domains.

Thus, $\vdash$ is a binary relation over $L$ and, as such, some of its basic properties may be noted. First and most obvious, $\vdash$ is an incomplete relation over $L$; i.e., for arbitrary $l, l^{\prime} \in L$, it may be that neither $l \vdash l^{\prime}$ nor $l^{\prime} \vdash l$ holds. Second, $\vdash$ respects so-called von Neumann-Morgensten independence; i.e. with the usual notation for convex combinations of lotteries, for any $l, l^{\prime}, l^{\prime \prime} \in L$ and $\alpha \in(0,1], l \vdash l^{\prime}$ if and only if $\alpha l+(1-\alpha) l^{\prime \prime} \vdash \alpha l^{\prime}+(1-\alpha) l^{\prime \prime}$.

\footnotetext{
${ }^{3}$ The variation consists in the final addition "with at least one strict inequality in each direction." This permits avoiding to say, e.g., that a lottery is riskier than its worst payoff. Given the comparative context in which we study spreads, the variation is stylistic only.

${ }^{4}$ Transposed to lotteries to render the two definitions comparable, Grant and Quiggin's (2005, Def. 1) notion of increasing uncertainty is a particular case of our notion of increasing risk. Grant and Quiggin's notion then requires that the inter-quantile differences increase (in absolute value) away from the crossing point. Our notion does not require that inter-quantile differences be defined or, even when they are, that they be so constrained.
} 
Finally, $\vdash$ is not necessarily transitive. ${ }^{5}$ Nevertheless, when desired, transitive sub-relations of $\vdash$ may be defined as follows (see Bommier et al., 2012, p. 1627-1628). Given $r \in(0,1)$, say that $l$ is a $r$-spread of $l^{\prime}$, noted here $l \vdash_{r} l^{\prime}$, if there exists a $k, 2 \leq k \leq n$, such that for all $i \leq k-1, r \geq$ $\sum_{j=1}^{i} p_{j} \geq \sum_{j=1}^{i} q_{j}$, and for all $i \geq k, r \leq \sum_{j=1}^{i} p_{j} \leq \sum_{j=1}^{i} q_{j}$. Notice that, given two lotteries $l$ and $l^{\prime}$ defined over finitely many payoffs, $l \vdash_{r} l^{\prime}$ and $l \vdash{ }_{r^{\prime}} l^{\prime}$ may simultaneously hold for distinct $r, r^{\prime} \in(0,1) .{ }^{6}$ However, whether uniqueness holds or not, each $\vdash_{r}$ relation is transitive.

An example of the just introduced $r$-spread relations is with $r=1 / 2$, which corresponds to the special case of a median-preserving spread (Allison and Foster, 2004; Lasso de la Vega, 2018). Given other values of $r$, other quantile-preserving spreads can be similarly defined (Mendelson, 1987; without this terminology but with a similar idea, Bommier et al., 2012). By definition, if $l$ is a basic spread of $l^{\prime}$, then it is a $r$-spread of $l^{\prime}$ for a given (range of) $r \in(0,1)$, which implies that it is a spread of $l^{\prime}$, while none of the converse implications holds. This delineates a map of various degrees of increase in risk over finite domains. This map could be enriched-but to a limited extent only, especially since following the present approach, the associated cumulative distribution functions must single-cross. ${ }^{7}$

\section{Risk Aversion}

Relative to the notions of increasing risk introduced in the previous section, concepts of absolute risk attitude could certainly be defined. However, these concepts would prove uninteresting, inasmuch as they would clash with core properties of decision-making under risk. ${ }^{8}$ Accordingly, what one should examine instead is the more flexible and more primitive notion of a comparative risk attitude. To that end, consider decision-makers 1 and 2, endowed with binary preference relations $\succcurlyeq_{1}$ and $\succcurlyeq_{2}$, respectively, over $L$. More specifi-

\footnotetext{
${ }^{5}$ For illustration, take $X=\{a, b, c, d, e\}$. Assume that the set is ordered alphabetically. Consider the lotteries $l=(2 / 5, a ; 0, b ; 2 / 5, c ; 0, d ; 1 / 5, e), l^{\prime}=(1 / 5, a ; 0, b ; 0, c ; 4 / 5, d ; 0, e)$, and $l^{\prime \prime}=(0, a ; 3 / 5, b ; 0, c ; 2 / 5, d ; 0, e)$. Then $l$ is a spread of $l^{\prime}$ and $l^{\prime}$ is a spread of $l^{\prime \prime}$, but $l$ is not a spread of $l^{\prime \prime}$ because the cumulative distribution functions of $l$ and $l^{\prime \prime}$ multiple-cross.

${ }^{6}$ Take the alphabetically ordered set $X=\{a, b, c\}$ and consider the lotteries $l=(1 / 3, a ; 1 / 3, b ; 1 / 3, c)$ and $l^{\prime}=(1 / 4, a ; 1 / 2, b ; 1 / 4, c)$. Then, $l \vdash_{r} l^{\prime}$ for all $r \in[1 / 3,2 / 3]$.

${ }^{7}$ While this seems to prevent one from generalizing the notion of a spread, in the other direction, some special kinds of $r$-spread may very well be defined. Consider, for example, the variance order (Trojani and Malamud, 2009). In effect, it is defined with reference to $1 / 2$-spreads, with the added restriction that, for any function $u: X \rightarrow \mathbb{R}$ representing the restriction of $\succcurlyeq$ to $X$, the variance of $u$ is greater in the riskier lottery than in the less risky one. (Surprisingly perhaps, some such comparisons are indeed ordinally robust.)

${ }^{8}$ For instance, given some $r \in(0,1)$, say that $\succcurlyeq$ is averse to all $r$-spreads if for any $l, l^{\prime} \in L$, if $l \vdash_{r} l^{\prime}$, then $l^{\prime} \succcurlyeq l$. Assuming mixture-continuity (a standard property satisfied by RDU and many other models), one can show that this attitude clashes with the strict respect of first-order stochastic dominance. Similar conclusions obtain varying the kind of spread under consideration, or replacing risk aversion by risk seeking or risk neutrality.
} 
cally, assume throughout that $\succcurlyeq_{1}$ and $\succcurlyeq_{2}$ coincide over $X$, i.e., they differ over non-degenerate lotteries only. When it will be necessary to repeat this assumption, to be discussed shortly, we will refer to it as the assumption that $\succcurlyeq_{1}$ and $\succcurlyeq_{2}$ are ordinally equivalent. Notice that under the ordinal equivalence assumption, there is no need to index spread relations by either $\succcurlyeq_{1}$ or $\succcurlyeq_{2}$. This is because the notion of first-order stochastic dominance, hence the notion of single-crossing, are entirely ordinal notions; i.e., they are robust to any strictly increasing transformation of the underlying utility function on the set of payoffs. Equipped with this observation, consider the following definitions. For greater generality, they are given with reference not to $r$-spreads, for some $r \in(0,1)$, but to any spread. ${ }^{9}$

Definition 2. Let $\succcurlyeq_{1}$ and $\succcurlyeq_{2}$ be two ordinally equivalent decision-makers. Say that $\succcurlyeq_{1}$ is strongly more risk averse (respectively: seeking) than $\succcurlyeq_{2}$, noted here 1 SMRA 2 (respectively: 1 SMRS 2), if for all $l, l^{\prime} \in L$ such that $l \vdash l^{\prime}$, if $l \succcurlyeq_{1} l^{\prime}$, then $l \succcurlyeq_{2} l^{\prime}$ (respectively: if $l^{\prime} \succcurlyeq_{1} l$, then $l^{\prime} \succcurlyeq_{2} l$ ). Say that $\succcurlyeq_{1}$ is weakly more risk averse (respectively: seeking) than $\succcurlyeq_{2}$, noted here 1 WMRA 2 (respectively: 1 WMRS 2), if the previous implication holds under the additional condition that $l^{\prime}$ is degenerate, i.e., the spread is basic.

Thus, generally speaking, 1 is more risk averse (respectively: seeking) than 2 if there is no increase (respectively: decrease) in risk which 1 would accept but 2 refuse. ${ }^{10}$ The "weak" or "strong" qualification then applies depending on the restrictiveness of the kind of spread considered. For brevity, we henceforth focus on comparative risk aversion only; i.e., we forego expliciting the parallel statements pertaining to comparative risk seeking.

Notice that by definition, 1 SMRA 2 implies 1 WMRA 2, but the converse does not hold in general. This is a simple, but important fact to which we will return in the next section. We record it in the following statement.

Fact 1. Necessarily, if 1 SMRA 2, then 1 WMRA 2. The converse does not necessarily hold.

To the best of our knowledge, there is, in the current literature, no established terminology to describe various degrees of comparative risk aversion. The terminology which we propose is natural in light of the implication just highlighted. It is also aligned with the terminology already established to describe the various degrees of absolute risk aversion over classic convex real domains (see, e.g., Chateauneuf et al., 1997). Finally, speaking of "weak" and "strong" comparative risk aversion to describe the specific attitudes previously defined is justified by the observations made in Sec. 3. To wit, basic

\footnotetext{
${ }^{9}$ From now on, the notion of $r$-spread, by contrast with the more general notion of a spread, will not play any role in our paper. Our results will show that, under RDU at least, focusing on a specific $r$-spread is not relevant for analyzing comparative risk attitudes.

${ }^{10}$ Hence Yaari's "acceptance set" terminology (Yaari, 1969, for instance on p. 316).
} 
spreads seem to be the most restrictive, and spreads, the most inclusive, degrees of increase in risk which one could define over finite domains.

We close this section by briefly discussing the ordinal equivalence assumption. This assumption is typically considered necessary to meaningfully compare risk attitudes across individuals (e.g., Kihlstrom and Mirman, 1974, p. 366; Bommier et al., 2012, p. 1620). ${ }^{11}$ This assessment could be qualified, however. First, we observe that, in some cases, the addition of this assumption can be spared. Assume that, restricted to $X, \succcurlyeq_{2}$ is known to be a strict order. If 1 WMRA 2 holds, it then follows that $\succcurlyeq_{1}$ is also strict, furthermore that it coincides with $\succcurlyeq_{2}$, over $X$. Thus, in particular, if $\succcurlyeq_{1}$ and $\succcurlyeq_{2}$ are comparable in terms of risk attitudes and strict over $X$, they are ordinally equivalent. Second and more important, we observe that the ordinal equivalence assumption could be naturally generalized as follows. Say that $\succcurlyeq_{1}$ and $\succcurlyeq_{2}$ are permutation-ordinally equivalent if they are identical over $X$ up to some permutation on $X$. The permutation ordinal equivalence condition fails when $\succcurlyeq_{1}$ and $\succcurlyeq_{2}$ do not have the same number of indifference classes over $X$. When the condition obtains, however, the previous notions of comparative risk aversion can apply, provided they are generalized as explained next. Let $\sigma_{1}^{2}: X \rightarrow X$ be any permutation such that the permutation ordinal equivalence condition holds. ${ }^{12}$ Furthermore, abusing notation, for any $l \in L$, let $\sigma_{1}^{2}(l)$ denote the lottery induced by applying the permutation $\sigma_{1}^{2}$ to the payoffs of $l$. Finally, let $l \vdash^{\succcurlyeq} l^{\prime}$ denote the fact that $l$ is a spread of $l^{\prime}$ with respect to $\succcurlyeq_{i}$, with $i=1,2$. Then, notice that $l \vdash{ }_{1} l^{\prime}$ holds if and only if $\sigma_{1}^{2}(l) \vdash^{\succcurlyeq 2} \sigma_{1}^{2}\left(l^{\prime}\right)$ holds, with the crossing point being the same in both cases. Accordingly, we would find it natural to say, for example, that 1 is strongly more risk averse than 2 if for all $l, l^{\prime} \in L$ such that $l \vdash^{\succcurlyeq} l^{\prime}$, if $l \succcurlyeq_{1} l^{\prime}$, then $\sigma_{1}^{2}(l) \succcurlyeq_{2} \sigma_{1}^{2}\left(l^{\prime}\right)$. This illustrates the fact that risk attitude comparisons are not, in principle, bound by the ordinal equivalence assumption.

\section{Characterizations}

We now turn to the comparison of two RDU decision-makers, $\succcurlyeq_{1}$ and $\succcurlyeq_{2}$, characterized by the pairs of functions $\left(u_{1}, w_{1}\right)$ and $\left(u_{2}, w_{2}\right)$, respectively. To this end, we introduce the following two inter-individual indices. In the second index, owing to the ordinal equivalence assumption, we forego defining the domain over which the supremum is taken with specific reference to either $\succcurlyeq_{1}$ or $\succcurlyeq_{2}$, and we write $\succcurlyeq$ instead. $^{13}$

\footnotetext{
${ }^{11}$ Absent special assumptions, it does not follow from 1 WMRA 2 that 1 and 2 are ordinally equivalent. Indeed, 1 WMRA 2 can hold with, for instance, $\succcurlyeq_{2}$ the constant relation such that $x \sim_{2} y$ for all $x, y \in X$ and $\succcurlyeq_{1}$ any non-constant preference relation over $X$.

${ }^{12}$ When indifference between payoffs is allowed, the permutation is not uniquely defined.

${ }^{13}$ Further notice the use, in this index, of the assumption that there are at least three non-indifferent payoffs. Without this assumption, $w$ in (1) is not behaviorally identified anyhow (e.g., Gilboa, 1987, p. 71).
} 


$$
\begin{aligned}
& I_{w}^{1,2}=\inf _{\{p, q, r, s \in[0,1] \mid p>q \geq r>s\}} \frac{\frac{w_{1}(p)-w_{1}(q)}{w_{2}(p)-w_{2}(q)}}{\frac{w_{1}(r)-w_{1}(s)}{w_{2}(r)-w_{2}(s)}} . \\
& I_{u}^{1,2}=\sup _{\{a, b, c, d \in X \mid a \succ b \succcurlyeq c \succ d\}} \frac{\frac{u_{1}(a)-u_{1}(b)}{u_{2}(a)-u_{2}(b)}}{\frac{u_{1}(c)-u_{1}(d)}{u_{2}(c)-u_{2}(d)}} .
\end{aligned}
$$

To interpret these indices, observe first that, by the definition of RDU, there must exist a strictly increasing function $g_{1}: \mathbb{R} \rightarrow \mathbb{R}$ such that $w_{1}=g_{1} \circ w_{2}$. Likewise, by the additional assumption that $\succcurlyeq_{1}$ and $\succcurlyeq_{2}$ are ordinally equivalent, there must exist a strictly increasing function $h_{1}: \mathbb{R} \rightarrow \mathbb{R}$ such that $u_{1}=h_{1} \circ u_{2}$. Therefore, using these increasing transformations $g_{1}$ and $h_{1}$, one could also introduce $I_{w}^{1,2}$ and $I_{u}^{1,2}$ as follows.

$$
\begin{gathered}
I_{w}^{1,2}=\inf _{\{p, q, r, s \in[0,1] \mid p>q \geq r>s\}} \frac{\frac{g_{1}\left(w_{2}(p)\right)-g_{1}\left(w_{2}(q)\right)}{w_{2}(p)-w_{2}(q)}}{\frac{g_{1}\left(w_{2}(r)\right)-g_{1}\left(w_{2}(s)\right)}{w_{2}(r)-w_{2}(s)}} . \\
I_{u}^{1,2}=\sup _{\{a, b, c, d \in X \mid a \succ b \succcurlyeq c \succ d\}} \frac{\frac{h_{1}\left(u_{2}(a)\right)-h_{1}\left(u_{2}(b)\right)}{u_{2}(a)-u_{2}(b)}}{\frac{h_{1}\left(u_{2}(c)\right)-h_{1}\left(u_{2}(d)\right)}{u_{2}(c)-u_{2}(d)}} .
\end{gathered}
$$

These variants make the following facts apparent. The minimand in (2) is a discrete and multiplicative analogue of a second derivative value for $w_{1}$ as a function of (the range of) $w_{2}$. Therefore, $I_{w}^{1,2}$ is an index of non-convexity of $w_{1}$ as a function of $w_{2}$. The lower the value of the index, the less convex the function. Similarly, the maximand in (3) is a discrete and multiplicative analogue of a second derivative value for $u_{1}$ as a function of (the range of) $u_{2}$. Thus, $I_{u}^{1,2}$ is an index of non-concavity of $u_{1}$ as a function of $u_{2}$. The higher the value of the index, the less concave the function. Notice that $u_{1}$ is more concave than $u_{2}$ if and only if $I_{u}^{1,2} \leq 1$. Furthermore, it can be shown that $w_{1}$ is more convex than $w_{2}$ if and only if $I_{w}^{1,2}=1$. This is because one can show that $I_{w}^{1,2} \leq 1$ always holds. This can be derived either from the fact that the range of the weighting functions is convex, or from the fact that their domain is convex (while by contrast, with a finite domain of payoffs, neither the domain nor the range of the utility functions is convex). We record the resulting equivalences in the following observation.

Observation 1. 1) $u_{1}$ is more concave than $u_{2}$ if and only if $I_{u}^{1,2} \leq 1$. 2) $w_{1}$ is more convex than $w_{2}$ if and only if $I_{w}^{1,2}=1$.

Proof. See the Appendix. 
The two inter-individual indices $I_{w}^{1,2}$ and $I_{u}^{1,2}$ are inspired by, and can be compared to, the two intra-individual indices introduced in Chateauneuf et al., 2005 to characterize so-called "monotone risk aversion". This is a specific degree of absolute risk aversion which one can define when the domain of payoffs is a convex subset of the reals. Instrumental in Chateauneuf et al.'s characterization of that attitude are the following indices $P_{w}$ and $G_{u}$, called by them indices of "pessimism" and "greediness", respectively.

$$
\begin{gathered}
P_{w}=\inf _{\{p \in(0,1)\}} \frac{\frac{1-w(p)}{1-p}}{\frac{w(p)}{p}} . \\
G_{u}=\sup _{\{a, b, c, d \in X \mid a>b \geq c>d\} \frac{u(a)-u(b)}{a-b}} \frac{\frac{u(c)-u(d)}{c-d}}{} .
\end{gathered}
$$

As can be seen by re-interpreting the domain of $u_{1}$ as the range of $u_{2}$, there is no fundamental difference between $I_{u}^{1,2}$ and $G_{u}$. By contrast, even after a similar transposition has been made, a relevant difference between $I_{w}^{1,2}$ and $P_{w}$ remains. To wit, define the following index of "relative pessimism".

$$
P_{w}^{1,2}=\inf _{\{p \in(0,1)\}} \frac{\frac{1-w_{1}(p)}{1-w_{2}(p)}}{\frac{w_{1}(p)}{w_{2}(p)}} .
$$

As comparing (2) and (6) makes clear, $P_{w}^{1,2}$ corresponds to the particular case of $I_{w}^{1,2}$ where the minimand is taken assuming $p=1, q=r$, and $s=0$ while $I_{w}^{1,2}$ ranges, more generally, over any $p, q, r, s \in[0,1]$ such that $p>q \geq r>s$. Accordingly, given functions $w_{1}$ and $w_{2}, I_{w}^{1,2} \leq P_{w}^{1,2}$ obviously always holds, while $I_{w}^{1,2}<P_{w}^{1,2}$ demonstrably holds in some cases. We will later return to this observation to illustrate the fact that the condition $P_{w}^{1,2} \geq I_{u}^{1,2}-$ which would be the direct comparative transposition of Chateauneuf et al.'s characterization - would not be strong enough to characterize SMRA under RDU (see esp. Obs. 3 and Ex. 3). ${ }^{14}$ The above differences notwithstanding, like $P_{w}$ and $G_{u}$ in Chateauneuf et al., 2005, $I_{w}^{1,2}$ and $I_{u}^{1,2}$ can be interpreted as indices of pessimism and greediness, respectively — only, as inter-individual, relative ones.

We can now state our main result.

\footnotetext{
${ }^{14}$ By contrast, in their characterization of their notion of comparative uncertainty aversion under Choquet Expected Utility, Grant and Quiggin (2005, Prop. 5) can use a comparative transposition of Chateauneuf et al.'s characterization. Before a referee draw our attention to Grant and Quiggin's paper, we were unaware that the conditions in Chateauneuf et al., 2005 had already been put to use to investigate general notions of comparative risk or uncertainty aversion. Grant and Quiggin's precedence notwithstanding, our main result cannot be obtained as a corollary of theirs (see Obs. 3 and Ex. 3).
} 
Theorem 1. Let $\succcurlyeq_{1}$ and $\succcurlyeq_{2}$ be two ordinally equivalent $R D U$ decisionmakers, characterized by the pairs of functions $\left(u_{1}, w_{1}\right)$ and $\left(u_{2}, w_{2}\right)$, respectively. Then, 1 SMRA 2 if and only if $I_{w}^{1,2} \geq I_{u}^{1,2}$.

Proof. See the Appendix.

Next, notice that if $w_{1}=w_{2}$ (respectively: $u_{1}=u_{2}$ ), then $I_{w}^{1,2}=1$ (respectively: $I_{u}^{1,2}=1$ ). Besides, recall that $I_{u}^{1,2} \leq 1$ if and only if $u_{1}$ is more concave than $u_{2}$, that $I_{w}^{1,2} \leq 1$ always holds, and that $I_{w}^{1,2}=1$ if and only if $w_{1}$ is more convex than $w_{2}$. Thus, Thm. 1 has as immediate corollaries the following two results. Inasmuch as they pertain to finite domains, they are also new, to the best of our knowledge.

Corollary 1. Let $\succcurlyeq_{1}$ and $\succcurlyeq_{2}$ be two ordinally equivalent EU decision-makers, characterized by the functions $u_{1}$ and $u_{2}$, respectively. Then, 1 SMRA 2 if and only if $u_{1}$ is more concave than $u_{2}$.

Corollary 2. Assuming that the elements of $X$ are real numbers, let $\succcurlyeq_{1}$ and $\succcurlyeq_{2}$ be two DEU decision-makers, characterized by the functions $w_{1}$ and $w_{2}$, respectively. Alternatively, with general $X$, let $\succcurlyeq_{1}$ and $\succcurlyeq_{2}$ be two $R D U$ decision-makers, characterized by the pairs of functions $\left(u_{1}, w_{1}\right)$ and $\left(u_{2}, w_{2}\right)$, respectively, with $u_{1}=u_{2}$ (up to some affine transformation). Then, 1 SMRA 2 if and only if $w_{1}$ is more convex than $w_{2}$.

It is worth examining what becomes of the above characterizations in the convex case, thereby meaning either that (i) the payoffs are numerical, their domain is convex, and the utility functions are strictly increasing over that domain; or that (ii) the range of the utility functions is convex. While neither sense of convexity implies the other, either suffices to derive the following corollary. The corollary holds because on either sense of convexity, $I_{u}^{1,2} \geq 1$ always holds (which is like in Chateauneuf et al., 2005, e.g. p. 658, and following the same reasoning as the one explained with respect to $I_{w}^{1,2}$ in the proof of Obs. 1). It then follows that, in the convex case, $u_{1}$ is more concave than $u_{2}$ if and only if $I_{u}^{1,2}=1$.

Corollary 3. Let $\succcurlyeq_{1}$ and $\succcurlyeq_{2}$ be two ordinally equivalent $R D U$ decisionmakers, characterized by the pairs of functions $\left(u_{1}, w_{1}\right)$ and $\left(u_{2}, w_{2}\right)$, respectively. Furthermore, let either the range of $u_{1}$ and $u_{2}$ be convex, or the payoffs be numerical, their domain be convex, and the utility functions $u_{1}$ and $u_{2}$ be strictly increasing over that domain. Then, 1 SMRA 2 if and only if $u_{1}$ is more concave than $u_{2}$ and $w_{1}$ is more convex than $w_{2}$.

Corr. 3 also implies that, in the convex case, the characterizations of SMRA under EU and DEU remain the same as the ones given in Corrs. 1 and 2, re- 
spectively. ${ }^{15}$ On the assumption that the utility functions have convex range, more specifically, the three preceding results can also be obtained as corollaries of the classic characterizations of the absolute concept of "strong risk aversion" (i.e., aversion to all mean-preserving spreads; see, e.g., Chateauneuf et al., 1997). ${ }^{16}$ See, for EU, Rothschild and Stiglitz, 1970; for DEU, Yaari, 1987, Thm. 2; for RDU (under various technical restrictions), Chew et al. 1987, Thm. 1, Ebert, 2004, Thm. 4, and Schmidt and Zank, 2008, Thm. 1.

Thus, under either EU or DEU, SMRA is characterized by the same condition in the finite and the convex case. However, interestingly, the same conclusion does not hold under general RDU. Now, under RDU, for 1 SMRA 2 to hold, it is necessary in the finite case, like it is in the convex case, that $u_{1}$ be more concave than $u_{2}$. Indeed, $I_{w}^{1,2} \leq 1$ always holds and $I_{u}^{1,2} \leq 1$ if and only if $u_{1}$ is more concave than $u_{2}$, so that $I_{w}^{1,2} \geq I_{u}^{1,2}$ together with non-concavity would lead to a contradiction. But in the finite case, unlike in the convex case, $w_{1}$ need not be more convex than $w_{2}$ for 1 SMRA 2 to hold. Indeed, when $X$ is finite, it can even be under RDU that 1 SMRA 2 and $w_{1}$ is strictly more concave than $w_{2}$. This is established by the following example.

Example 1. Let $X=\{1,4 / 9,1 / 9\}$. For all $x \in X$, let $u_{2}(x)=x$ and $u_{1}(x)=\sqrt{x}$. For all $p \in[0,1]$, let $w_{2}(p)=p$ and $w_{1}(p)=9 / 8 p-1 / 8 p^{2}$, as illustrated graphically in Figure $1 .{ }^{17}$

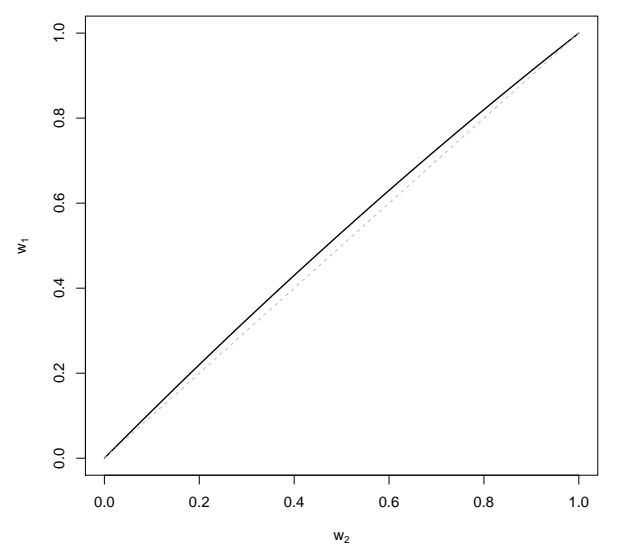

Figure 1: $w_{1}(p)=\frac{9}{8} p-\frac{1}{8} p^{2}$, strictly more concave than $w_{2}(p)=p$

\footnotetext{
${ }^{15}$ For alternative proofs of these characterization results under EU and DEU, see Bommier et al., 2012, Results 3.1 and 3.2, respectively. The fact that Bommier et al.'s results pertain to the convex case follows from how they model the larger intertemporal decision problem in which they embed their characterization exercises (see esp. p. 1617). These modelling assumptions also explain why their Result 3.2 is a theorem about DEU.

${ }^{16}$ The corollaries hold because the fact stated next follows from Thm. 1 in Machina and Pratt, 1997 (perfecting Thm. 1 in Rothschild and Stiglitz, 1970). In the case of RDU preferences (among others), aversion to single-crossing mean-preserving spreads is equivalent to aversion to all mean-preserving spreads (multiple-crossing ones included).

${ }^{17}$ More generally, for any $\lambda \in(1,5 / 4)$, one could take $w_{1}(p)=\lambda p+(1-\lambda) p^{2}$ here.
} 
Thus, $u_{1}$ is strictly more concave than $u_{2}$. As $w_{1}{ }^{\prime}(p)=9 / 8-2 / 8 p \geq \frac{7}{8}>0$ and $w_{1}^{\prime \prime}(p)=-2 / 8<0$, it also holds that $w_{1}$ is strictly more concave than $w_{2}$. But $I_{u}^{1,2}=\frac{\frac{1-2 / 3}{1-4 / 9}}{\frac{2 / 3-1 / 3}{4 / 9-1 / 9}}=3 / 5$ while $I_{w}^{1,2}=\inf _{1>p \geq q>0} \frac{w_{1}{ }^{\prime}(p)}{w_{1}{ }^{\prime}(q)}=\frac{7 / 8}{9 / 8}=7 / 9$, so that $I_{w}^{1,2}>I_{u}^{1,2}$. By Thm. 1, 1 SMRA 2 .

Next, we consider the weakening of SMRA to WMRA. The characterization of WMRA under general RDU is a difficult problem on which we have little progress to report. We observe that this characterization is an open question even in the convex case. More specifically, even on the assumption that the utility functions have convex range, providing the result would require characterizing under RDU the absolute concept of "weak risk aversion" (i.e., aversion to mean-preserving spreads with the added condition that the less risky lottery is degenerate). This is a longstanding open problem of the field (see Chateauneuf and Cohen, 1994; Chateauneuf et al., 1997).

However, as we now explain in several steps, interesting conclusions can be reached nonetheless. First, as a baseline observation, we recall the characterization of WMRA under EU. It is proved for arbitrary domains as Thm. 2 in Peters and Wakker, $1987 .{ }^{18}$

Theorem 2 (Peters and Wakker). Let $\succcurlyeq_{1}$ and $\succcurlyeq_{2}$ be two ordinally equivalent EU decision-makers, characterized by the functions $u_{1}$ and $u_{2}$, respectively. Then, 1 WMRA 2 if and only if $u_{1}$ is more concave than $u_{2}$.

Next, we consider DEU. However preliminary to a characterization under DEU of WMRA over finite domains, the following result - the crux of which is not the sufficiency, but the non-necessity part - is new. It seems to have been previously conjectured (Köbberling and Peters, 2003, Lemma 2.2), but not proved, to the best of our knowledge.

Observation 2. Assuming that the elements of $X$ are real numbers and $X$ is a finite set, let $\succcurlyeq_{1}$ and $\succcurlyeq_{2}$ be two DEU decision-makers, characterized by the functions $w_{1}$ and $w_{2}$, respectively. Alternatively, with general $X$, let $\succcurlyeq_{1}$ and $\succcurlyeq_{2}$ be two RDU decision-makers, characterized by the pairs of functions $\left(u_{1}, w_{1}\right)$ and $\left(u_{2}, w_{2}\right)$, respectively, with $u_{1}=u_{2}$ (up to some affine transformation) and $u_{1}, u_{2}$ finite-ranged. Then, for 1 WMRA 2 to hold, it is sufficient but not necessary that for all $p \in[0,1], w_{1}(p) \leq w_{2}(p)$.

\footnotetext{
${ }^{18}$ While the result given next states that under ordinal equivalence, 1 WMRA 2 holds under EU if and only if $u_{1}$ is a strictly increasing concave function of $u_{2}$, Peters and Wakker show that, absent any assumption about ordinal equivalence or the lack thereof, 1 WMRA 2 holds under EU if and only if $u_{1}$ is a non-decreasing concave function of $u_{2}$.
} 
Proof. Sufficiency is obvious from (11) and the observation that a spread is basic if the spread conditions hold, for some $k$, with $\sum_{j=1}^{i} q_{j}=0$ for all $i \leq k-1$ and $\sum_{j=1}^{i} q_{j}=1$ for all $i \geq k$. Non-necessity is established by the following example.

Example 2. Let $X=\{1,1 / 2,0\}$. For all $x \in X$, let $u_{1}(x)=u_{2}(x)=x$. For all $p \in[0,1]$, let $w_{2}(p)=p$ and $w_{1}(p)$ be defined as follows: ${ }^{19}$

$$
w_{1}(p)= \begin{cases}\frac{5}{4} p & \text { if } p \in\left[0, \frac{2}{10}\right] \\ \frac{5}{7} p+\frac{3}{28} & \text { if } p \in\left(\frac{2}{10}, \frac{9}{10}\right) . \\ \frac{5}{2} p-\frac{3}{2} & \text { if } p \in\left[\frac{9}{10}, 1\right]\end{cases}
$$

This is illustrated graphically in Figure 2.

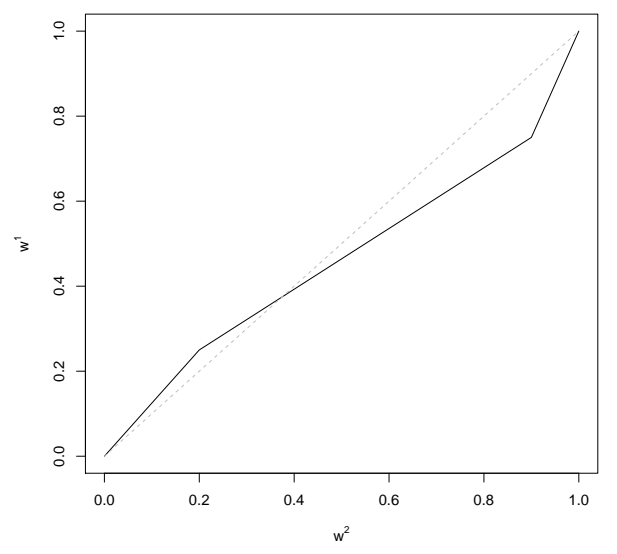

Figure 2: $w_{1}(p)$ defined as in $(7)$, with $w_{1}(p)>w_{2}(p)$ for all $p \in\left(0, \frac{3}{8}\right)$.

Then, we have that $w_{1}(p)<w_{2}(p)$ for all $p \in\left(\frac{3}{8}, 1\right)$ but $w_{1}(p)>w_{2}(p)$ for all $p \in\left(0, \frac{3}{8}\right)$. Although $w_{1}(p) \leq w_{2}(p)$ thus does not hold for all $p \in[0,1]$, it still holds that 1 WMRA 2, as we show in the Appendix.

\footnotetext{
${ }^{19}$ More generally, with any $\alpha \in(0,1 / 2), \beta \in(1 / 2,1)$ such that $\alpha+\beta>1$, one could here define $w_{1}$ as the polygon through the points $(0,0),(\alpha, 1 / 4),(\beta, 3 / 4)$, and $(1,1)$, i.e.:$$
w_{1}(p)= \begin{cases}\frac{p}{4 \alpha} & \text { if } p \in[0, \alpha] \\ \frac{1}{4}+\frac{p-\alpha}{2(\beta-\alpha)} & \text { if } p \in(\alpha, \beta) . \\ \frac{3}{4}+\frac{p-\beta}{4(1-\beta)} & \text { if } p \in[\beta, 1]\end{cases}
$$ 
The full characterization of WMRA under DEU is a problem which we leave open and hope to solve in future work.

Finally, consider general RDU. However preliminary, the following resultthe crux of which is not the non-necessity, but the sufficiency part-also seems new, and its relevance will be seen shortly. It builds on insights in Chateauneuf and Cohen, 1994 and Chateauneuf et al., 2005, respectively.

Observation 3. Let $\succcurlyeq_{1}$ and $\succcurlyeq_{2}$ be two ordinally equivalent $R D U$ decisionmakers, characterized by the pairs of functions $\left(u_{1}, w_{1}\right)$ and $\left(u_{2}, w_{2}\right)$, respectively. Then, for 1 WMRA 2 to hold, it is sufficient but not necessary that $P_{w}^{1,2} \geq I_{u}^{1,2}$ and that for all $p \in[0,1], w_{1}(p) \leq w_{2}(p)$.

Proof. Non-necessity is obvious from Ex. 2, since 1 WMRA 2 holds but $w_{1}(p) \leq w_{2}(p)$ for all $p \in[0,1]$ does not. Sufficiency is proved in the Appendix.

Once again, the preceding results about WMRA are preliminary. As we now explain, they are instructive nonetheless. First, observe that in the convex case, the following characterizations obtain. Under EU, it still holds that 1 WMRA 2 if and only if $u_{1}$ is more concave than $u_{2}$. (This is because Peters and Wakker's result has no domain restriction; assuming $u_{1}$ and $u_{2}$ convex-ranged, more specifically, the result could also be obtained as a corollary of the classic characterization of absolute weak risk aversion.) Under DEU, 1 WMRA 2 if and only if, for all $p \in[0,1], w_{1}(p) \leq w_{2}(p)$. (This is, on either construal of convexity, a corollary of the characterization of absolute weak risk aversion; see Quiggin, 1991, Prop. 1, Köbberling and Peters, 2003, Lemma 2.2, as well as Yaari, 1986.) Thus, under EU, WMRA is characterized identically across the finite and the convex cases. However, the same conclusion does not hold under DEU, hence, under RDU more generally. This much can be claimed even though, once again, the full characterizations of WMRA under RDU and under DEU are still unknown.

Second, one should put side by side Cor. 1 and Thm. 2 on EU, and Cor. 2 and Obs. 2 on DEU together with Thm. 1 and Obs. 3 on general RDU. Recall Fact 1 to the effect that 1 SMRA 2 implies 1 WMRA 2 by definition, while the converse does not hold in general. But under EU, the converse implication does hold, i.e., 1 WMRA 2 implies 1 SMRA 2. By contrast, under DEU or under general RDU, the converse implication does not hold, i.e., it can be that 1 WMRA 2 but not 1 SMRA 2. As a first example, take any pair of DEU decision-makers with probability weighting functions $w_{1}$ and $w_{2}$, respectively, such that i) for all $p \in[0,1], w_{1}(p) \leq w_{2}(p)$ ii) $w_{1}$ is not more convex than $w_{2}$. Under DEU, condition i) and the negation of condition ii) are respectively sufficient for WMRA and necessary for SMRA, so that 1 WMRA 2 but not 1 SMRA 2. As another example, take any 
pair of RDU decision-makers characterized by the pairs of functions $\left(u_{1}, w_{1}\right)$ and $\left(u_{2}, w_{2}\right)$, respectively, such that i) $P_{w}^{1,2} \geq I_{u}^{1,2}$ and for all $p \in[0,1]$, $w_{1}(p) \leq w_{2}(p)$ ii) $I_{w}^{1,2}<I_{u}^{1,2}$. Under RDU, condition i) and the negation of condition ii) are respectively sufficient for WMRA and necessary for SMRA, so that once again, 1 WMRA 2 but not 1 SMRA 2. The following concrete example illustrates both possibilities at once.

Example 3. Let $X=\{4.5,4.1,3.1,3,2.1,2,1.1,1,0.5,0\} .{ }^{20}$ For all $p \in[0,1]$, let $w_{2}(p)=p$ and $w_{1}(p)$ be defined as follows:

$$
w_{1}(p)= \begin{cases}p(1-p) & \text { if } p \in\left[0, \frac{1}{2}\right] \\ 3\left(p-\frac{1}{2}\right)^{2}+\frac{1}{4} & \text { if } p \in\left(\frac{1}{2}, 1\right]\end{cases}
$$

This is illustrated graphically in Figure 3.

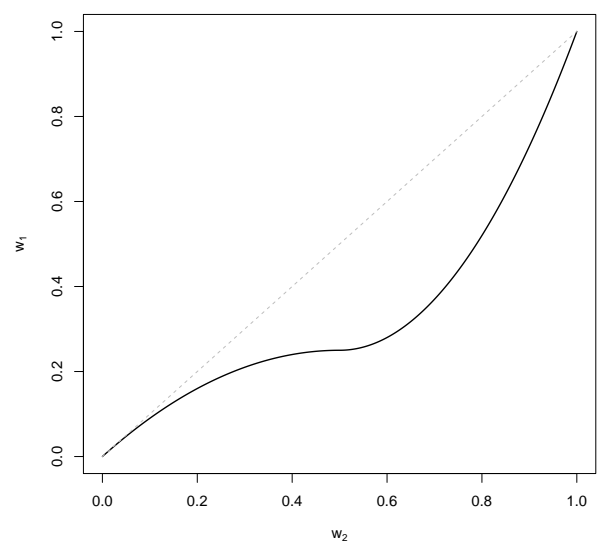

Figure 3: $w_{1}(p)$ defined as in (8).

In the DEU variant of the example, let $u_{1}(x)=u_{2}(x)=x$. Given that $w_{2}(p) \leq w_{1}(p)$ for all $p \in[0,1]$ but $w_{2}$ is strictly more concave than $w_{1}$ over $[0,1 / 2]$, we have by Cor. 2 and Obs. 2 that 1 WMRA 2 but not 1 SMRA 2. To verify the latter, take lotteries $l=(1 / 8,4.5 ; 1 / 8,4 ; 1 / 8,2 ; 1 / 8,0.5 ; 1 / 2,0)$ and $l^{\prime}=(1 / 8,4.1 ; 1 / 8,3.1 ; 1 / 8,2.1 ; 1 / 8,1.1 ; 1 / 2,0) . \quad$ As $v_{1}(l)=\frac{53}{64}>\frac{50}{64}=v_{1}\left(l^{\prime}\right)$ but $v_{2}(l)=\frac{25}{20}<\frac{26}{20}=v_{2}\left(l^{\prime}\right)$, we have that $l \succ_{1} l^{\prime}$ but $l \prec_{2} l^{\prime}$. Thus, since $l \vdash l^{\prime}$, it is not the case that 1 SMRA 2.

\footnotetext{
${ }^{20}$ Both variants of the example would deliver the same conclusions if one took, instead of this finite set $X$, its convex hull. The DEU variant would then apply without any change, while the only change in the RDU variant would consist in that $I_{u}^{1,2}=1$.
} 
In the general $R D U$ variant of the example, let $u_{1}(x)=\sqrt{x}$ and $u_{2}(x)=x$. Then, it can be checked that $I_{u}^{1,2}=\frac{\frac{u_{1}(4.1)-u_{1}(4)}{u_{2}(4.1)-u_{2}(4)}}{\frac{u_{1}(4)-u_{1}(3.1)}{u_{2}(4)-u_{2}(3.1)}}=9 \frac{(\sqrt{4.1}-2)}{(2-\sqrt{3.1})} \approx 0.9$, that $P_{w}^{1,2}=1\left(P_{w}^{1,2} \geq 1\right.$ holds since $w_{1}(p) \leq w_{2}(p)$ for all $p \in[0,1]$, and $P_{w}^{1,2} \leq 1$ holds since $\left.w_{1}^{\prime}(0)=1\right)$, and that $I_{w}^{1,2}=0$ ( since $\left.w_{1}^{\prime}\left(\frac{1}{2}\right)=0\right)$. Thus it holds that $P_{w}^{1,2}>I_{u}^{1,2}>I_{w}^{1,2}$, so that by Thm. 1 and Obs. 3, 1 WMRA 2 but not $1 S M R A$ 2. To verify the latter, take again the lotteries $l$ and $l^{\prime}$ previously specified. As $v_{1}(l) \approx 0.445>0.443 \approx v_{1}\left(l^{\prime}\right)$ but $v_{2}(l)=\frac{25}{20}<\frac{26}{20}=v_{2}\left(l^{\prime}\right)$, we have that $l \succ_{1} l^{\prime}$ but $l \prec_{2} l^{\prime}$. Thus, since $l \vdash l^{\prime}$, it is not the case that 1 SMRA 2.

Thus, we can now state synthetically how our study improves on the current state of the literature on the treatment of risk attitudes in EU and RDU, respectively. First, from classic results on absolute risk attitudes over convex domains of real payoffs, it was already known that, unlike RDU, EU imposes what can be called the "strengthening of risk attitudes" (Chateauneuf et al., 1997, 2004; Baccelli, 2018). For example, under EU, unlike under RDU, a decision-maker cannot be weakly, yet not strongly, risk averse. More or less directly, it then follows that, in the convex case, a similar conclusion holds true of the strengthening of comparative risk attitudes. Thus, what our study adds to the current state of the literature in this respect is that, when the domain is finite, the comparative variant of the strengthening conclusion still holds. Indeed, even when the utility functions have finite range, under EU, it cannot be that 1 WMRA 2 but not 1 SMRA 2, while this is possible under RDU. We record this fact in the following statement.

Fact 2. When the utility functions have finite range, under EU, WMRA and $S M R A$ are characterized by the same condition. Under $R D U$, such is not the case.

Second, we have also shown that there is a previously unnoticed dimension along which the different ways in which EU and RDU treat risk attitudes can be analyzed. To wit, we have introduced the question of whether the same condition characterizes WMRA (respectively: SMRA) in the finite and the convex case (convexity referring here either to the domain, or to the range, of the utility functions). As we have shown, the characterizing condition is the same under EU (viz., greater concavity), but not under RDU. Recall indeed that under general RDU, in the convex case, 1 SMRA 2 holds if and only if $u_{1}$ is more concave than $u_{2}$ and $w_{1}$ is more convex than $w_{2}$, but that the latter condition is unnecessary in the finite case (see Ex. 1). As another example, under the special DEU case of RDU, in the convex case, 1 WMRA 2 holds if and only if $w_{1}(p) \leq w_{2}(p)$ for all $p \in[0,1]$, but this condition is unnecessary in the finite case (see Ex. 2). Thus, notwithstanding the fact that the complete characterization of WMRA under RDU (or even 
its special DEU case) is currently open question, the findings above can be summarized as stated next.

Fact 3. Under EU, WMRA (respectively: SMRA) is characterized by the same condition in the finite and the convex case. Under RDU, such is not the case.

Admittedly, fully appreciating Fact 3-including how its interpretation should compare to that of the more straightforward Fact 2-will demand more work. But it is in any case relevant evidence for understanding the structural differences between EU and RDU in their respective treatment of risk attitudes.

\section{Conclusion}

We have investigated notions of weak and strong comparative risk aversion that are applicable even over a finite, non-numerical domain of payoffs. We have shown that under expected utility, weak and strong comparative risk aversion are characterized by the same condition not only when the compared utility functions have convex range, which was already known, but also when they have finite range, which had not been hitherto established. We have also shown that, under expected utility still, weak (respectively: strong) comparative risk aversion is characterized by the same condition in the finite and the convex case, thus introducing a new kind of comparisons to the literature. As we have explained by contrast in the rank-dependent utility model, neither of the above conclusions needs to hold under non-expected utility.

As we now illustrate by singling out four possible directions for future research, our study of risk aversion over finite domains thus raises several interesting further questions. First, we have left open how to fully characterize weak comparative risk aversion in the dual expected utility model. This task should be more manageable than, and preparatory to, the much more difficult task of characterizing that attitude under general rank-dependent utility. As previously noted, the latter problem is tightly connected to a famous open question of the field. Given the results already available, solving either of these problems would improve on our current understanding of the divide between expected and non-expected utility. Second, we have focused exclusively on either the finite or the convex case. However, it would also be worth investigating intermediary cases, such as the case of utility functions with a countable range. Through the characterization of weak and strong comparative risk aversion, such intermediary cases could lead to a more nuanced understanding of the structural properties of the main models of decision-making under risk. Third, we have focused exclusively on rankdependent utility preferences. One natural next step would be to consider the larger class of "smooth" non-expected utility preferences, for which characterizations of comparative risk aversion are available in the convex-ranged 
case (Machina, 1982, Sec. 3.3; Cerreia-Vioglio et al., 2016, Sec. 4). An interesting first task would be, then, to determine how these characterizations should be generalized in the finite-ranged case. Finally, equipped with the more expressive notions of comparative risk aversion discussed in our paper, it would be worth revisiting some of the comparative statics exercises already presented in the literature. For instance, building on Bommier et al., 2012 and Köbberling and Peters, 2003, respectively, the notions we have examined here could provide new insights on classic comparative statics topics in the economics of saving behavior or in bargaining theory. Other comparative statics analysis could be similarly refined.

\section{Appendix}

\section{Proof of Observation 1}

Proof. The proof of the first claim is obvious, so we focus on the second one. First, if $I_{w}^{1,2}=1$, then $w_{1}$ is more convex than $w_{2}$. Second, if $w_{1}$ is more convex than $w_{2}$, then $I_{w}^{1,2} \geq 1$. But, as we now show, $I_{w}^{1,2} \leq 1$ always holds. More specifically, as we now explain, this fact can be proved either from the convexity of the range of the weighting functions, or from the convexity of their domain.

Consider first the proof from the convexity of the range of the weighting functions. ${ }^{21}$ We start by observing that by the defining properties of weighting functions, the function $w_{1} \circ\left(w_{2}\right)^{-1}$ is well defined. Next, since $w_{2}$ has a convex range, the composition $w_{1} \circ\left(w_{2}\right)^{-1}$ has a convex domain. Additionally, since $w_{1}$ and $w_{2}$ are strictly increasing, $w_{1} \circ\left(w_{2}\right)^{-1}$ also has this property. Thus, by the variant of the Lebesgue Differentiability Theorem pertaining to increasing functions, the transformation $w_{1} \circ\left(w_{2}\right)^{-1}$ has at least one point of differentiability. If this point has zero derivative, then $I_{w}^{1,2}=0 \leq 1$. If this point has non-zero derivative, then it cannot be the case that $I_{w}^{1,2}>1$, for using its four degrees of freedom, the minimand can then be made arbitrary close to 1 . In all cases, then, $I_{w}^{1,2} \leq 1$.

Consider next the proof from the convexity of the domain of the weighting functions. Since $w_{2}$ is a strictly increasing function over a convex domain, by the same variant of the Lebesgue Differentiability Theorem as in the preceding paragraph, $w_{2}$ has at least one point $p$ of differentiability. This implies in particular that $w_{2}$ has, with $p$, at least one point of continuity. This in turn means that there is a neighbourhood $(p-\varepsilon, p+\varepsilon)$ where $w_{2}$ is a strictly increasing continuous function. Thus, due to the Intermediate Value Theorem, the restriction of $w_{2}$ to that neighbourhood has a convex range.

\footnotetext{
${ }^{21}$ Since weighting functions are defined and strictly increasing over [0,1], the convexity of the range of $w_{1}$ and $w_{2}$ is equivalent to $w_{1}$ and $w_{2}$ being continuous over their domain. As regards the adaptation of the above arguments to prove Cor. 3 , notice that a utility function can be convex-ranged and nevertheless be defined over a non-numerical domain.
} 
Based on this observation, the proof for the convex-domained case can be reduced to the proof for the convex-ranged case by replacing $w_{1} \circ\left(w_{2}\right)^{-1}$ by $w_{1} \circ\left(\tilde{w}_{2}\right)^{-1}$, with $\tilde{w}_{2}$ the restriction of $w_{2}$ to the neighbourhood $(p-\varepsilon, p+\varepsilon)$.

\section{Proof of Theorem 1}

$\underline{I_{w}^{1,2} \geq I_{u}^{1,2} \Rightarrow 1 \text { SMRA } 2 .}$

Proof. Assume $I_{w}^{1,2} \geq I_{u}^{1,2}$. We need to show that if $l \vdash l^{\prime}$ and $l \succcurlyeq_{1} l^{\prime}$, then $l \succcurlyeq_{2} l^{\prime}$. Let $l=\left(p_{1}, x_{1} ; \ldots ; p_{n}, x_{n}\right)$ and $l^{\prime}=\left(q_{1}, x_{1} ; \ldots ; q_{n}, x_{n}\right)$. Under RDU, $l \succcurlyeq_{1} l^{\prime}$ if and only if:

$$
\sum_{i=1}^{n}\left[\left(w_{1}\left(\sum_{j=1}^{i} p_{j}\right)-w_{1}\left(\sum_{j=1}^{i} q_{j}\right)\right)\left(u_{1}\left(x_{i}\right)-u_{1}\left(x_{i+1}\right)\right)\right] \geq 0 .
$$

Define an index $i$ in (9) as null if either $u_{1}\left(x_{i}\right)=u_{1}\left(x_{i+1}\right)$ or $\sum_{j=1}^{i} p_{j}=\sum_{j=1}^{i} q_{j}$, and as non-null otherwise. Eliminate all null indices in (9) and let $I$ be the set of all non-null indices. ${ }^{22}$ Thus, (9) reads as follows:

$$
\sum_{i \in I}\left[\left(w_{1}\left(\sum_{j=1}^{i} p_{j}\right)-w_{1}\left(\sum_{j=1}^{i} q_{j}\right)\right)\left(u_{1}\left(x_{i}\right)-u_{1}\left(x_{i+1}\right)\right)\right] \geq 0
$$

with the sum in (10) featuring only non-null terms.

Now, by definition, $l \vdash l^{\prime}$ if and only if for some $k$ with $2 \leq k \leq n$, for all $i \leq k-1, \sum_{j=1}^{i} p_{j} \geq \sum_{j=1}^{i} q_{j}$, and for all $i \geq k, \sum_{j=1}^{i} p_{j} \leq \sum_{j=1}^{i} q_{j}$, with at least one strict inequality in each direction. Accordingly, let $I^{+}$(resp. $I^{-}$) be the set of all non-null indices with $\sum_{j=1}^{i} p_{j} \geq \sum_{i=1}^{i} q_{j}$ (resp. $\sum_{j=1}^{i} p_{j} \leq \sum_{i=1}^{i} q_{j}$ ). Thus, (10) holds if and only if:

\footnotetext{
${ }^{22}$ Notice that $n$ must be a null index, since $\sum_{j=1}^{n} p_{j}=\sum_{j=1}^{n} q_{j}=1$. Further notice that if all indices are null, then $l \sim_{1} l^{\prime}$ and by the definition of the weighting function or the ordinal equivalence assumption, $l \sim_{2} l^{\prime}$ holds as well, which trivially establishes the claim. Accordingly, one may make the non-triviality assumption that $I$ is not empty.
} 


$$
\begin{aligned}
\sum_{i \in I^{+}}[ & \left.\left(w_{1}\left(\sum_{j=1}^{i} p_{j}\right)-w_{1}\left(\sum_{j=1}^{i} q_{j}\right)\right)\left(u_{1}\left(x_{i}\right)-u_{1}\left(x_{i+1}\right)\right)\right] \geq \\
\sum_{i \in I^{-}} & {\left[\left(w_{1}\left(\sum_{j=1}^{i} q_{j}\right)-w_{1}\left(\sum_{j=1}^{i} p_{j}\right)\right)\left(u_{1}\left(x_{i}\right)-u_{1}\left(x_{i+1}\right)\right)\right], }
\end{aligned}
$$

with only strictly positive terms, if any, on both sides of the inequality sign in (11).

Next, let $B$ be:

$$
B=\prod_{i \in I} \frac{w_{2}\left(\sum_{j=1}^{i} p_{j}\right)-w_{2}\left(\sum_{j=1}^{i} q_{j}\right)}{w_{1}\left(\sum_{j=1}^{i} p_{j}\right)-w_{1}\left(\sum_{j=1}^{i} q_{j}\right)} \prod_{i \in I} \frac{u_{2}\left(x_{i}\right)-u_{2}\left(x_{i+1}\right)}{u_{1}\left(x_{i}\right)-u_{1}\left(x_{i+1}\right)}
$$

noticing that:

$$
\prod_{i \in I^{-}} \frac{w_{2}\left(\sum_{j=1}^{i} p_{j}\right)-w_{2}\left(\sum_{j=1}^{i} q_{j}\right)}{w_{1}\left(\sum_{j=1}^{i} p_{j}\right)-w_{1}\left(\sum_{j=1}^{i} q_{j}\right)}=\prod_{i \in I^{-}} \frac{w_{2}\left(\sum_{j=1}^{i} q_{j}\right)-w_{2}\left(\sum_{j=1}^{i} p_{j}\right)}{w_{1}\left(\sum_{j=1}^{i} q_{j}\right)-w_{1}\left(\sum_{j=1}^{i} p_{j}\right)} .
$$

Similarly, assuming that $I$ is not a singleton, ${ }^{23}$ let $B_{-l}$ be:

$$
B_{-l}=\prod_{i \in I \backslash\{l\}} \frac{w_{2}\left(\sum_{j=1}^{i} p_{j}\right)-w_{2}\left(\sum_{j=1}^{i} q_{j}\right)}{w_{1}\left(\sum_{j=1}^{i} p_{j}\right)-w_{1}\left(\sum_{j=1}^{i} q_{j}\right)} \prod_{i \in I \backslash\{l\}} \frac{u_{2}\left(x_{i}\right)-u_{2}\left(x_{i+1}\right)}{u_{1}\left(x_{i}\right)-u_{1}\left(x_{i+1}\right)}
$$

Since all $i \in I$ are non-null and the ordinal equivalence assumption holds, $B>0$ and similarly, $B_{-l}>0$ for all $l \in I$. Thus, (11) holds if and only if:

\footnotetext{
${ }^{23}$ If $I$ is a singleton, then it must be that the only non-null index is in $I^{+}$, for otherwise the assumption $l \succcurlyeq_{1} l^{\prime}$ would be violated. When there is only one non-null index, multiplying by $B$ both sides in inequality (11) directly establishes the claim.
} 


$$
\begin{gathered}
B \sum_{i \in I^{+}}\left[\left(w_{1}\left(\sum_{j=1}^{i} p_{j}\right)-w_{1}\left(\sum_{j=1}^{i} q_{j}\right)\right)\left(u_{1}\left(x_{i}\right)-u_{1}\left(x_{i+1}\right)\right)\right] \geq \\
B \sum_{i \in I^{-}}\left[\left(w_{1}\left(\sum_{j=1}^{i} q_{j}\right)-w_{1}\left(\sum_{j=1}^{i} p_{j}\right)\right)\left(u_{1}\left(x_{i}\right)-u_{1}\left(x_{i+1}\right)\right)\right] \\
\Leftrightarrow \quad \sum_{i \in I^{+}} B_{-i}\left[\left(w_{2}\left(\sum_{j=1}^{i} p_{j}\right)-w_{2}\left(\sum_{j=1}^{i} q_{j}\right)\right)\left(u_{2}\left(x_{i}\right)-u_{2}\left(x_{i+1}\right)\right)\right] \geq \\
\sum_{i \in I^{-}} B_{-i}\left[\left(w_{2}\left(\sum_{j=1}^{i} q_{j}\right)-w_{2}\left(\sum_{j=1}^{i} p_{j}\right)\right)\left(u_{2}\left(x_{i}\right)-u_{2}\left(x_{i+1}\right)\right)\right] .
\end{gathered}
$$

Next, observe that $I_{w}^{1,2} \geq I_{u}^{1,2}$ can be rewritten equivalently as:

$$
\inf _{\{p, q, r, s \in[0,1] \mid p>q \geq r>s\}} \frac{\frac{w_{2}(r)-w_{2}(s)}{w_{1}(r)-w_{1}(s)}}{\frac{w_{2}(p)-w_{2}(q)}{w_{1}(p)-w_{1}(q)}} \geq \sup _{\{a, b, c, d \in X \mid a \succ b \succcurlyeq c \succ d\}} \frac{\frac{u_{2}(c)-u_{1}(d)}{u_{1}(c)-u_{1}(d)}}{\frac{u_{2}(a)-u_{2}(b)}{u_{1}(a)-u_{1}(b)}}
$$

Thus, $I_{w}^{1,2} \geq I_{u}^{1,2}$ implies that for any $i \in I$,

$$
\frac{w_{2}\left(\sum_{j=1}^{i-1} p_{j}\right)-w_{2}\left(\sum_{j=1}^{i-1} q_{j}\right)}{w_{1}\left(\sum_{j=1}^{i-1} p_{j}\right)-w_{1}\left(\sum_{j=1}^{i-1} q_{j}\right)} \frac{u_{2}\left(x_{i-1}\right)-u_{2}\left(x_{i}\right)}{u_{1}\left(x_{i-1}\right)-u_{1}\left(x_{i}\right)} \geq \frac{w_{2}\left(\sum_{j=1}^{i} p_{j}\right)-w_{2}\left(\sum_{j=1}^{i} q_{j}\right)}{w_{1}\left(\sum_{j=1}^{i} p_{j}\right)-w_{1}\left(\sum_{j=1}^{i} q_{j}\right)} \frac{u_{2}\left(x_{i}\right)-u_{2}\left(x_{i+1}\right)}{u_{1}\left(x_{i}\right)-u_{1}\left(x_{i+1}\right)}
$$

Thus, $I_{w}^{1,2} \geq I_{u}^{1,2}$ implies that $B_{-l}$ increases in $l$. Accordingly, with $i^{*}$ the last index in $I^{+}$and $l^{*}$ the first index in $I^{-}$, we have that for all $i \in I^{+}$, $B_{-i} \leq B_{-i^{*}}$ and for all $i \in I^{-}, B_{-i} \geq B_{-l^{*}}$. Consequently, together with $I_{w}^{1,2} \geq I_{u}^{1,2},(14)$ implies:

$$
\begin{gathered}
B_{-i^{*}} \sum_{i \in I^{+}}\left[\left(w_{2}\left(\sum_{j=1}^{i} p_{j}\right)-w_{2}\left(\sum_{j=1}^{i} q_{j}\right)\right)\left(u_{2}\left(x_{i}\right)-u_{2}\left(x_{i+1}\right)\right)\right] \geq \\
B_{-l^{*}} \sum_{i \in I^{-}}\left[\left(w_{2}\left(\sum_{j=1}^{i} q_{j}\right)-w_{2}\left(\sum_{j=1}^{i} p_{j}\right)\right)\left(u_{2}\left(x_{i}\right)-u_{2}\left(x_{i+1}\right)\right)\right] .
\end{gathered}
$$


Since $B_{-l}>0$ for all $l \in I$ and $B_{-l}$ increases in $l$, we have that $\frac{B_{-i^{*}}}{B_{-l^{*}}}$ is well defined and $\frac{B_{-i^{*}}}{B_{-l^{*}}} \leq 1$, so that (15) implies:

$$
\begin{aligned}
\sum_{i \in I^{+}}[ & \left.\left(w_{2}\left(\sum_{j=1}^{i} p_{j}\right)-w_{2}\left(\sum_{j=1}^{i} q_{j}\right)\right)\left(u_{2}\left(x_{i}\right)-u_{2}\left(x_{i+1}\right)\right)\right] \geq \\
\sum_{i \in I^{-}} & {\left[\left(w_{2}\left(\sum_{j=1}^{i} q_{j}\right)-w_{2}\left(\sum_{j=1}^{i} p_{j}\right)\right)\left(u_{2}\left(x_{i}\right)-u_{2}\left(x_{i+1}\right)\right)\right] . }
\end{aligned}
$$

Reestablishing the indices null in (9) and rearranging the equation, we thus obtain from (16):

$$
\sum_{i=1}^{n}\left[\left(w_{2}\left(\sum_{j=1}^{i} p_{j}\right)-w_{2}\left(\sum_{j=1}^{i} q_{j}\right)\right)\left(u_{2}\left(x_{i}\right)-u_{2}\left(x_{i+1}\right)\right)\right] \geq 0
$$

which, under RDU, holds if and only if $l \succcurlyeq_{2} l^{\prime}$, as was to be shown.

$\underline{1 \text { SMRA } 2 \Rightarrow I_{w}^{1,2} \geq I_{u}^{1,2} \text {. }}$

Proof. Recalling our assumption that there are at least three non-indifferent payoffs, consider any $a, b, c, d \in X$ such that $u_{1}(a)>u_{1}(b) \geq u_{1}(c)>u_{1}(d)$. Take any $p, s \in[0,1]$ such that $p>s$. Our first step is the observation that by the continuity of $w_{1}$, there exists $q, r \in(p, s)$ such that $q \geq r$ and:

$$
\frac{w_{1}(r)-w_{1}(s)}{w_{1}(p)-w_{1}(q)}=\frac{u_{1}(c)-u_{1}(d)}{u_{1}(a)-u_{1}(b)}
$$

Our second step is the observation that for any $x, y, z \in X$ such that $u_{1}(x) \geq u_{1}(a)>u_{1}(b) \geq u_{1}(y) \geq u_{1}(c)>u_{1}(d) \geq u_{1}(z)$, with $p>q \geq r>s$, $l, l^{\prime} \in L$ given by $l=(s, x ;(r-s), a ;(q-r), y ;(p-q), d ;(1-p), z)$ and $l^{\prime}=(s, x ;(r-s), b ;(q-r), y ;(p-q), c ;(1-p), z)$ are such that $l \vdash l^{\prime}$. Besides, under RDU, (18) holds if and only if $l \sim_{1} l^{\prime}$. As 1 SMRA 2, it then follows that $l \succcurlyeq_{2} l^{\prime}$. Under RDU, $l \succcurlyeq_{2} l^{\prime}$ holds if and only if:

$$
\frac{w_{2}(r)-w_{2}(s)}{w_{2}(p)-w_{2}(q)} \geq \frac{u_{2}(c)-u_{2}(d)}{u_{2}(a)-u_{2}(b)}
$$

Combining inequality (19) and equality (18), we thus have that for those particular $a \succ_{1} b \succcurlyeq_{1} c \succ_{1} d \in X$ and $p>q \geq r>s \in[0,1]$ : 


$$
\frac{\frac{w_{1}(p)-w_{1}(q)}{w_{2}(p)-w_{2}(q)}}{\frac{w_{1}(r)-w_{1}(s)}{w_{2}(r)-w_{2}(s)}} \geq \frac{\frac{u_{1}(a)-u_{1}(b)}{u_{2}(a)-u_{2}(b)}}{\frac{u_{1}(c)-u_{1}(d)}{u_{2}(c)-u_{2}(d)}}
$$

Next, call $\lambda$ the term on the right-hand side of the inequality in (18). Lemma 1 in Chateauneuf et al. (2005) can then be adapted to establish that:

$$
\inf _{\substack{\left\{p, q, r, s \in[0,1] \mid p>q \geq r>s \\ \frac{w_{1}(r)-w_{1}(s)}{w_{1}(p)-w_{1}(q)}=\lambda\right\}}} \frac{\frac{w_{1}(p)-w_{1}(q)}{w_{2}(p)-w_{2}(q)}}{\frac{w_{1}(r)-w_{1}(s)}{w_{2}(r)-w_{2}(s)}}=\inf _{\{p, q, r, s \in[0,1] \mid p>q \geq r>s\}} \frac{\frac{w_{1}(p)-w_{1}(q)}{\frac{w_{2}(p)-w_{2}(q)}{w_{1}(r)-w_{1}(s)}}}{w_{2}(r)-w_{2}(s)}
$$

Thus, (20) implies in particular that:

$$
\inf _{\{p, q, r, s \in[0,1] \mid p>q \geq r>s\}} \frac{\frac{w_{1}(p)-w_{1}(q)}{w_{2}(p)-w_{2}(q)}}{\frac{w_{1}(r)-w_{1}(s)}{w_{2}(r)-w_{2}(s)}} \geq \sup _{\left\{a, b, c, d \in X \mid a \succ_{1} b \succcurlyeq_{1} c \succ_{1} d\right\}} \frac{\frac{u_{1}(a)-u_{1}(b)}{u_{2}(a)-u_{2}(b)}}{\frac{u_{1}(c)-u_{1}(d)}{u_{2}(c)-u_{2}(d)}},
$$

which, by the ordinal equivalence assumption, establishes the claim.

\section{Proof of the claim in Example 2}

Proof. We need to show that under the assumptions of the example, 1 WMRA 2.

Notice that under these assumptions, under DEU:

1 WMRA $2 \Leftrightarrow\left(p_{1}, 1 ; p_{2}, 1 / 2, p_{3}, 0\right) \succcurlyeq_{1} 1 / 2 \Rightarrow\left(p_{1}, 1 ; p_{2}, 1 / 2, p_{3}, 0\right) \succcurlyeq_{2} 1 / 2$

$$
\begin{aligned}
& \Leftrightarrow w_{1}\left(p_{1}\right) \cdot \frac{1}{2}+w_{1}\left(p_{1}+p_{2}\right) \cdot \frac{1}{2} \geq \frac{1}{2} \Rightarrow w_{2}\left(p_{1}\right) \cdot \frac{1}{2}+w_{2}\left(p_{1}+p_{2}\right) \cdot \frac{1}{2} \geq \frac{1}{2} \\
& \Leftrightarrow w_{1}\left(p_{1}\right)+w_{1}\left(p_{1}+p_{2}\right) \geq 1 \Rightarrow 2 p_{1}+p_{2} \geq 1 .
\end{aligned}
$$

Next, case by case, one can check that $w_{1}$ is such that $w_{1}(p)+w_{1}(1-p)<1$ for all $p \in(0,1)$. First, for any $p \in\left(0, \frac{1}{10}\right]$, we have that $(1-p) \in\left[\frac{9}{10}, 1\right)$, which implies that $w_{1}(p)+w_{1}(1-p)=\frac{5}{4} p+\frac{5}{2}(1-p)-\frac{3}{2}=-\frac{5}{4} p+1<1$. Second, for any $p \in\left(\frac{1}{10}, \frac{2}{10}\right]$, we have that $(1-p) \in\left[\frac{8}{10}, \frac{9}{10}\right)$, which implies that $w_{1}(p)+w_{1}(1-p)=\frac{5}{4} p+\frac{5}{7}(1-p)+\frac{3}{28}=-\frac{15}{28} p+\frac{23}{28} \leq \frac{15}{28} \frac{2}{10}+\frac{23}{28}=\frac{6}{28}<1$. Finally, for any $p \in\left(\frac{2}{10}, \frac{1}{2}\right]$, we have that $(1-p) \in\left[\frac{1}{2}, \frac{8}{10}\right)$, which implies that $w_{1}(p)+w_{1}(1-p)=\frac{5}{7} p+\frac{3}{28}+\frac{5}{7}(1-p)+\frac{3}{28}=\frac{6}{28}+\frac{5}{7}=\frac{26}{28}<1$. All cases where $p>\frac{1}{2}$ can be checked analogously by switching the roles of $p$ and $(1-p)$ in the above arguments. Now, the fact that $w_{1}(p)+w_{1}(1-p)<1$ holds for all $p \in(0,1)$ ensures that 1 WMRA 2 holds. Indeed, this property, $w_{1}\left(p_{1}\right)+w_{1}\left(p_{1}+p_{2}\right) \geq 1$, and the fact that $w_{1}$ is increasing together imply that $p_{1}+p_{2}>1-p_{1}$, thus, that $2 p_{1}+p_{2} \geq 1$, as was to be shown. 


\section{Proof of Observation 3}

Proof. Assume $P_{w}^{1,2} \geq I_{u}^{1,2}$ and $w_{1}(p) \leq w_{2}(p)$ for all $p \in[0,1]$. We need to show that if $l \vdash l^{\prime}, l^{\prime}$ is riskless, and $l \succcurlyeq_{1} l^{\prime}$, then $l \succcurlyeq_{2} l^{\prime}$. The proof closely resembles that of the sufficiency direction of Thm. 1, so we state it more succinctly.

First, observe that if $l \vdash l^{\prime}$ and $l^{\prime}$ is riskless, then with the same notation for (and under the same qualifications regarding) non-null indices as in the proof of Thm. $1, l \succcurlyeq_{1} l^{\prime}$ implies:

$$
\begin{aligned}
\sum_{i \in I^{+}}\left[\left(w_{1}\left(\sum_{j=1}^{i} p_{j}\right)\right)\left(u_{1}\left(x_{i}\right)-u_{1}\left(x_{i+1}\right)\right)\right] \geq \\
\sum_{i \in I^{-}}\left[\left(1-w_{1}\left(\sum_{j=1}^{i} p_{j}\right)\right)\left(u_{1}\left(x_{i}\right)-u_{1}\left(x_{i+1}\right)\right)\right] .
\end{aligned}
$$

Next, let $B$ be:

$$
B=\prod_{i \in I^{+}} \frac{w_{2}\left(\sum_{j=1}^{i} p_{j}\right)}{w_{1}\left(\sum_{j=1}^{i} p_{j}\right)} \prod_{i \in I^{-}} \frac{1-w_{2}\left(\sum_{j=1}^{i} p_{j}\right)}{1-w_{1}\left(\sum_{j=1}^{i} p_{j}\right)} \prod_{i \in I} \frac{u_{2}\left(x_{i}\right)-u_{2}\left(x_{i+1}\right)}{u_{1}\left(x_{i}\right)-u_{1}\left(x_{i+1}\right)}
$$

with the necessary adaptations for $B_{-l}, l \in I$. Given that $B>0,(23)$ implies:

$$
\begin{gathered}
B \sum_{i \in I^{+}}\left[\left(w_{1}\left(\sum_{j=1}^{i} p_{j}\right)\right)\left(u_{1}\left(x_{i}\right)-u_{1}\left(x_{i+1}\right)\right)\right] \geq \\
B \sum_{i \in I^{-}}\left[\left(1-w_{1}\left(\sum_{j=1}^{i} p_{j}\right)\right)\left(u_{1}\left(x_{i}\right)-u_{1}\left(x_{i+1}\right)\right)\right] \\
\Leftrightarrow \quad \sum_{i \in I^{+}} B_{-i}\left[\left(w_{2}\left(\sum_{j=1}^{i} p_{j}\right)\right)\left(u_{2}\left(x_{i}\right)-u_{2}\left(x_{i+1}\right)\right)\right] \geq \\
\sum_{i \in I^{-}} B_{-i}\left[\left(1-w_{2}\left(\sum_{j=1}^{i} p_{j}\right)\right)\left(u_{2}\left(x_{i}\right)-u_{2}\left(x_{i+1}\right)\right)\right] .
\end{gathered}
$$


Now, let $i^{*} \in I^{+}$(resp. $l^{*} \in I^{-}$) be the index-or, in case of ties, one of the indices - such that for all $i \in I^{+}$(resp. $\left.i \in I^{-}\right), B_{-i} \leq B_{-i^{*}}\left(\right.$ resp. $\left.B_{-i} \geq B_{-l^{*}}\right)$. Thus, (25) implies:

$$
\begin{aligned}
B_{-i^{*}} \sum_{i \in I^{+}} & {\left[\left(w_{2}\left(\sum_{j=1}^{i} p_{j}\right)\right)\left(u_{2}\left(x_{i}\right)-u_{2}\left(x_{i+1}\right)\right)\right] \geq } \\
B_{-l^{*}} \sum_{i \in I^{-}} & {\left[\left(1-w_{2}\left(\sum_{j=1}^{i} p_{j}\right)\right)\left(u_{2}\left(x_{i}\right)-u_{2}\left(x_{i+1}\right)\right)\right] . }
\end{aligned}
$$

Next, as $w_{1}(p) \leq w_{2}(p)$ for all $p \in[0,1]$, one can show (see Chateauneuf et al., 2005, Prop. 2.(vi)) that the following equality holds:

$$
\inf _{\{p \in(0,1)\}} \frac{\frac{1-w_{1}(p)}{1-w_{2}(p)}}{\frac{w_{1}(p)}{w_{2}(p)}}=\inf _{\{p \geq q \in(0,1)\}} \frac{\frac{w_{2}(q)}{w_{1}(q)}}{\frac{1-w_{2}(p)}{1-w_{1}(p)}} .
$$

Accordingly, $P_{w}^{1,2} \geq I_{u}^{1,2}$ can be equivalently stated as follows:

$$
\inf _{\{p \geq q \in(0,1)\}} \frac{\frac{w_{2}(q)}{w_{1}(q)}}{\frac{1-w_{2}(p)}{1-w_{1}(p)}} \geq \sup _{\{a, b, c, d \in X \mid a \succ b \succcurlyeq c \succ d\}} \frac{\frac{u_{2}(c)-u_{1}(d)}{u_{1}(c)-u_{1}(d)}}{u_{2}(a)-u_{2}(b)} .
$$

Consequently, for any $i \in I^{+}, l \in I^{-}$, we have that:

$$
\frac{w_{2}\left(\sum_{j=1}^{i} p_{j}\right)}{w_{1}\left(\sum_{j=1}^{i} p_{j}\right)} \frac{u_{2}\left(x_{i}\right)-u_{2}\left(x_{i+1}\right)}{u_{1}\left(x_{i}\right)-u_{1}\left(x_{i+1}\right)} \geq \frac{1-w_{2}\left(\sum_{j=1}^{l} q_{j}\right)}{1-w_{1}\left(\sum_{j=1}^{l} q_{j}\right)} \frac{u_{2}\left(x_{l}\right)-u_{2}\left(x_{l+1}\right)}{u_{1}\left(x_{l}\right)-u_{1}\left(x_{l+1}\right)} .
$$

Thus, we have that $\frac{B_{-i^{*}}}{B_{-l^{*}}} \leq 1$, so that (26) implies:

$$
\begin{aligned}
\sum_{i \in I^{+}}\left[\left(w_{2}\left(\sum_{j=1}^{i} p_{j}\right)\right)\left(u_{2}\left(x_{i}\right)-u_{2}\left(x_{i+1}\right)\right)\right] \geq \\
\sum_{i \in I^{-}}\left[\left(1-w_{2}\left(\sum_{j=1}^{i} p_{j}\right)\right)\left(u_{2}\left(x_{i}\right)-u_{2}\left(x_{i+1}\right)\right)\right] .
\end{aligned}
$$

Reestablishing the null indices and rearranging the equation, we thus obtain that $l \succcurlyeq_{2} l^{\prime}$, as was to be shown. 


\section{References}

Allison, R. and J. Foster (2004). Measuring Health Inequality Using Qualitative Data. Journal of Health Economics 23(3), 505-524.

Baccelli, J. (2018). Risk Attitudes in Axiomatic Decision Theory-A Conceptual Perspective. Theory and Decision 84(1), 61-82.

Bommier, A., A. Chassagnon, and F. Le Grand (2012). Comparative Risk Aversion: A Formal Approach with Applications to Saving Behavior. Journal of Economic Theory 147(4), 1614-1641.

Cerreia-Vioglio, S., F. Maccheroni, and M. Marinacci (2016). Stochastic Dominance Analysis without the Independence Axiom. Management Science 63(4), 1097-1109.

Chateauneuf, A. and M. Cohen (1994). Risk Seeking with Diminishing Marginal Utility in a Non-Expected Utility Model. Journal of Risk and Uncertainty 9(1), 77-91.

Chateauneuf, A., M. Cohen, and I. Meilijson (1997). New Tools to Better Model Behavior under Risk and Uncertainty-An Overview. Finance 18(1), 25-46.

Chateauneuf, A., M. Cohen, and I. Meilijson (2004). Four Notions of Mean-Preserving Increase in Risk, Risk Attitudes and Applications to the Rank-Dependent Expected Utility Model. Journal of Mathematical Economics 40(5), 547-571.

Chateauneuf, A., M. Cohen, and I. Meilijson (2005). More Pessimism than Greediness: A Characterization of Monotone Risk Aversion in the RankDependent Expected Utility Model. Economic Theory 25(3), 649-667.

Chew, S. H., E. Karni, and Z. Safra (1987). Risk Aversion in the Theory of Expected Utility with Rank Dependent Probabilities. Journal of Economic Theory 42(2), 370-381.

Diamond, P. and J. Stiglitz (1974). Increases in Risk and in Risk Aversion. Journal of Economic Theory 8(3), 337-360.

Ebert, U. (2004). Social Welfare, Inequality, and Poverty When Needs Differ. Social Choice and Welfare 23(3), 415-448.

Eeckhoudt, L., C. Gollier, and H. Schlesinger (2005). Economic and Financial Decisions under Risk. Princeton: Princeton University Press.

Gilboa, I. (1987). Expected Utility with Purely Subjective Non-Additive Probabilities. Journal of Mathematical Economics 16(1), 65-88. 
Grant, S. and J. Quiggin (2005). Increasing uncertainty: a definition. Mathematical Social Sciences 49(2), 117-141.

Kihlstrom, R. and L. Mirman (1974). Risk Aversion with Many Commodities. Journal of Economic Theory 8(3), 361-388.

Köbberling, V. and H. Peters (2003). The Effect of Decision Weights in Bargaining Problems. Journal of Economic Theory 110(1), 154-175.

Lasso de la Vega, C. (2018). Social Inequality: Theoretical Approaches. In C. D'Ambrosio (Ed.), Handbook of Research on Economic and Social Well-Being, pp. 377-399. Cheltenham: Edward Elgar Publishing.

Machina, M. (1982). "Expected Utility" Analysis without the Independence Axiom. Econometrica 50(2), 277-323.

Machina, M. and J. Pratt (1997). Increasing Risk: Some Direct Constructions. Journal of Risk and Uncertainty 14(2), 103-127.

Mendelson, H. (1987). Quantile-Preserving Spread. Journal of Economic Theory 42(2), 334-351.

Peters, H. and P. Wakker (1987). Convex Functions on Non-Convex Domains. Economics Letters 22(2), 251-255.

Quiggin, J. (1982). A Theory of Anticipated Utility. Journal of Economic Behavior 83 Organization 3(4), 323-343.

Quiggin, J. (1991). Increasing Risk: Another Definition. In A. Chikán (Ed.), Progress in Decision, Utility and Risk Theory, pp. 239-248. Dordrecht: Springer.

Rothschild, M. and J. Stiglitz (1970). Increasing Risk: I. A Definition. Journal of Economic Theory 2(3), 225-243.

Schmidt, U. and H. Zank (2008). Risk Aversion in Cumulative Prospect Theory. Management Science 54(1), 208-216.

Trojani, F. and S. Malamud (2009). Variance Covariance Orders and Median Preserving Spreads. Swiss Finance Institute Research Paper.

Wakker, P. (2010). Prospect Theory-For Risk and Ambiguity. Cambridge: Cambridge University Press.

Yaari, M. (1969). Some Remarks on Measures of Risk Aversion and on their Uses. Journal of Economic Theory 1(3), 315-329. 
Yaari, M. (1986). Univariate and Multivariate Comparisons of Risk Aversion: A New Approach. In W. Heller, R. M. Starr, and D. A. Starrett (Eds.), Essays in honor of Kenneth J. Arrow, Volume III, pp. 173-187. Cambridge: Cambridge University Press.

Yaari, M. (1987). The Dual Theory of Choice under Risk. Econometrica 55(1), 95-115. 